MATHEMATICS OF COMPUTATION

Volume 77, Number 261, January 2008, Pages 159-179

S 0025-5718(07)02016-9

Article electronically published on July 26, 2007

\title{
EVALUATING THE EVANS FUNCTION: ORDER REDUCTION IN NUMERICAL METHODS
}

\author{
SIMON MALHAM AND JITSE NIESEN
}

\begin{abstract}
We consider the numerical evaluation of the Evans function, a Wronskian-like determinant that arises in the study of the stability of travelling waves. Constructing the Evans function involves matching the solutions of a linear ordinary differential equation depending on the spectral parameter. The problem becomes stiff as the spectral parameter grows. Consequently, the Gauss-Legendre method has previously been used for such problems; however more recently, methods based on the Magnus expansion have been proposed. Here we extensively examine the stiff regime for a general scalar Schrödinger operator. We show that although the fourth-order Magnus method suffers from order reduction, a fortunate cancellation when computing the Evans matching function means that fourth-order convergence in the end result is preserved. The Gauss-Legendre method does not suffer from order reduction, but it does not experience the cancellation either, and thus it has the same order of convergence in the end result. Finally we discuss the relative merits of both methods as spectral tools.
\end{abstract}

\section{INTRODUCTION}

Many partial differential equations admit travelling wave solutions; these are solutions that move at a constant speed without changing their shape. Such travelling waves occur in many fields, including biology, chemistry, fluid dynamics, and optics. It is often important to know whether a given travelling wave is stable: does it persist under small perturbations? A major step towards determining the stability of a travelling wave is to locate the spectrum of the linearization of the differential operator about the travelling wave. Evans [10] considers a shooting and matching method for this task. Evans introduced a function to measure the mismatch for a specific class of reaction-diffusion equations. This function was called the Evans function by Alexander, Gardner and Jones 2], who generalized its definition considerably. Since then, the Evans function has been used frequently for stability analysis; see, for example, [1, 3, 4, 6, 15] for numerical computations employing the Evans function and [11, 22, 30, 34, 35] for an analytic treatment. The review paper by Sandstede [33] gives an excellent overview of the field.

The Evans function is a function of one argument, the spectral parameter $\lambda$, and zeros of the Evans function correspond to eigenvalues of the corresponding operator. Hence, one can get information about the spectrum by finding zeros of

Received by the editor April 20, 2006 and, in revised form, November 15, 2006.

2000 Mathematics Subject Classification. Primary 65L15; Secondary 65L20, 65N25.

Key words and phrases. Evans function, Magnus method, order reduction.

This work was supported by EPSRC First Grant GR/S22134/01.

(C)2007 American Mathematical Society Reverts to public domain 28 years from publication 
the Evans function, either analytically or numerically. Our focus here is on the numerical approach.

The main part in the numerical evaluation of the Evans function is the solution of a linear ordinary differential equation depending on the spectral parameter $\lambda$. This is often done with an off-the-shelf integrator using an explicit Runge-Kutta method. Afendikov and Bridges 1 noticed that when $\lambda$ grows, the problem may become stiff, and therefore they use a Gauss-Legendre method. Recently, Aparicio, Malham and Oliver [3] proposed a new procedure based on the Magnus expansion, building on the work of Moan 25] and Greenberg and Marletta [14] who used the Magnus expansion to solve Sturm-Liouville problems. Aparicio et al. noticed that the Magnus method suffers from order reduction in the stiff regime. This means that the fourth-order integrators whose global error should scale like $h^{4}$ when the step size $h$ is small, instead converge more slowly. They analyzed this phenomenon in a modified Airy equation using the WKB-method. However, they restricted themselves to those values of $\lambda$ which correspond to the essential spectrum of the linearized differential operator.

The current paper continues the analysis of the Magnus method in the context of Evans function evaluations. We concentrate on scalar Schrödinger operators to simplify the analysis. Other methods based on a transformation to Prüfer variables 32 probably perform better in the scalar setting, but these methods cannot be used unchanged in the non-self-adjoint case where our interest lies.

We present another approach to the analysis of the Magnus method based on a power series expansion, which is valid for values of $\lambda$ outside the essential spectrum. We will show that the Magnus method also suffers from order reduction in this regime. Specifically, the relative local error is of order $\lambda^{-1 / 2} h^{2}$ as $h \rightarrow 0$ with $|\lambda|^{1 / 2} h \gg 1$. However, there are two subsequent important observations. First, when going from the local to the global error, one does not lose a factor of $h$ (as usual), but the global error is also of order $\lambda^{-1 / 2} h^{2}$. Second, the order reduction disappears completely when we evaluate the matching condition: the relative error in the Evans function is of order $\lambda^{-1 / 2} h^{4}$, thus quartic in the step size, just as one would expect from a fourth-order method. Since useful asymptotic estimates invoke an order $\lambda^{-1}$ error, at best, our numerical schemes even with order reduction prove a useful spectral tool in the regime $|\lambda| \ll h^{-8}$.

The phenomenon of order reduction was discovered for implicit Runge-Kutta methods by Prothero and Robinson [31. Nowadays, it is understood within the framework of B-convergence (see for instance [16, §IV.15]). The stability of Magnus methods has been analyzed for a highly-oscillatory equation by Iserles [19], for Schrödinger equations by Hochbruck and Lubich [17, and for parabolic equations by González, Ostermann and Thalhammer [13]. Unfortunately, these results cannot yet be fitted into a general theory 20 . The present paper can also be viewed as a contribution to this research.

We also present an analysis of the fourth-order Gauss-Legendre method. We show that the relative error committed by this method does not contain a term of order $\lambda^{-1 / 2} h^{2}$, but only smaller terms. Furthermore, the error decreases even further when evaluating the Evans function. As explained in more detail later, this is due to an effect similar to the one which makes the trapezoidal rule very efficient for the quadrature of periodic functions. 
The contents of this paper are as follows. In the next section, we define the Evans function and we give an asymptotic expression for the Evans function in the scalar case when the spectral parameter $\lambda$ is large in modulus and outside the essential spectrum. We then define the Magnus method in Section 3 . We show that the Magnus method suffers from order reduction, and we compute the error when evaluating the Evans function. We repeat the computation for the GaussLegendre method in the next section. The analysis is corroborated by numerical experiments in Section 5. In the final section, we compare our results with those of Aparicio, Malham and Oliver [3] and we discuss the stability of the Magnus method in general. More details of the intricate calculations presented in Sections 24 can be found in the technical report 29 .

\section{The Evans Function}

We are interested in homogeneous reaction-diffusion equations on an unbounded one-dimensional domain. Such equations have the form

$$
u_{t}=K u_{x x}+f(u)
$$

where $K$ is an $n$-by- $n$ diagonal matrix with positive entries (the diffusion coefficients) and the unknown $u$ is a function of $t$ and $x$. The function $f: \mathbf{R}^{n} \rightarrow \mathbf{R}^{n}$ describes the reaction term; we assume that $f$ is sufficiently smooth.

A travelling wave solution has the form $u(x, t)=\hat{u}(\xi)$ with $\xi=x-c t$ where $c$ is the wave speed; see for example Kolmogorov, Petrovsky and Piskunov [23]. We assume that a travelling wave solution for the equation is known, at least numerically. Furthermore, we assume that $\hat{u}$ is constant at infinity, meaning that the limits $\hat{u}_{ \pm}=\lim _{\xi \rightarrow \pm \infty} \hat{u}(\xi)$ exist (in fact, we will need later that additionally, the derivatives $\hat{u}^{(p)}$ vanish at infinity for $\left.p=1,2, \ldots\right)$. Such a wave is called a $p u l s e$ (if $\hat{u}_{+}=\hat{u}_{-}$) or a front (if $\hat{u}_{+} \neq \hat{u}_{-}$).

To study the stability of the travelling wave, we linearize (11) about the wave and write the result in the $(\xi, t)$ coordinate system which moves with the same speed as the travelling wave. This yields

$$
u_{t}=K u_{\xi \xi}+c u_{\xi}+D f(\hat{u}) u .
$$

Define the operator $\mathcal{L}$ by $\mathcal{L}(U)=K U^{\prime \prime}+c U^{\prime}+D f(\hat{u}) U$, where $U: \mathbf{R} \rightarrow \mathbf{C}^{n}$. Its spectrum determines whether (2) has solutions of the form $u(\xi, t)=\mathrm{e}^{\lambda t} U(\xi)$. The stability of the travelling wave $\hat{u}$ can be deduced from the location of the spectrum of $\mathcal{L}$.

The spectrum of $\mathcal{L}$ can be divided in two parts: the point spectrum $\sigma_{\mathrm{pt}}(\mathcal{L})$, consisting of those $\lambda \in \sigma(\mathcal{L})$ for which $\mathcal{L}-\lambda I$ is Fredholm of index zero 1 and the essential spectrum $\sigma_{\text {ess }}(\mathcal{L})$, which contains the rest of the spectrum. We assume that $\sigma_{\text {ess }}(\mathcal{L})$ is contained in the left half-plane $\{z \in \mathbf{C}: \operatorname{Re} z \leq 0\}$. This means that the spectral stability is determined by the position of the eigenvalues $\lambda \in \sigma_{\mathrm{pt}}(\mathcal{L})$.

We now introduce the Evans function, which is a tool for locating these eigenvalues. We rewrite the eigenvalue equation $\mathcal{L}(U)=\lambda U$ as the first-order differential equation

$$
\frac{\mathrm{d} y}{\mathrm{~d} \xi}=A(\xi ; \lambda) y
$$

\footnotetext{
${ }^{1} \mathrm{An}$ operator is Fredholm with index zero if its range is closed and the dimension of the null space equals the codimension of the range.
} 
where $y: \mathbf{R} \rightarrow \mathbf{C}^{2 n}$ and the matrix $A$ is given by

$$
A(\xi ; \lambda)=\left[\begin{array}{cc}
0 & I \\
K^{-1}(\lambda I-D f(\hat{u}(\xi))) & -c K^{-1}
\end{array}\right] .
$$

Since the spectral problem (3a) is a linear equation, its solutions form a linear space of dimension $2 n$. Define $E^{-}(\lambda)$ to be the subspace of solutions $y$ satisfying the boundary condition $y(\xi) \rightarrow 0$ as $\xi \rightarrow-\infty$. Similarly, $E^{+}(\lambda)$ denotes the subspace with $y(\xi) \rightarrow 0$ as $\xi \rightarrow+\infty$. Any eigenfunction must satisfy both boundary conditions and hence lie in the intersection of $E^{-}(\lambda)$ and $E^{+}(\lambda)$.

For all $\lambda \notin \sigma_{\text {ess }}(\mathcal{L})$, we have

$$
\operatorname{dim} E^{-}(\lambda)+\operatorname{dim} E^{+}(\lambda)=2 n .
$$

Choose a basis $y_{1}(\cdot ; \lambda), \ldots, y_{k}(\cdot ; \lambda)$ of $E^{-}(\lambda)$, where $k=\operatorname{dim} E^{-}(\lambda)$, and a basis $y_{k+1}(\cdot ; \lambda), \ldots, y_{2 n}(\cdot ; \lambda)$ of $E^{+}(\lambda)$. We can assemble these basis vectors, evaluated at an arbitrary point, say $\xi=0$, in the $2 n$-by- $2 n$ matrix

$$
\left[y_{1}(0 ; \lambda) \ldots y_{k}(0 ; \lambda) y_{k+1}(0 ; \lambda) \ldots y_{2 n}(0 ; \lambda)\right] .
$$

The Evans function, denoted $D(\lambda)$, is defined to be the determinant of this matrix. If the determinant vanishes, then the $y_{i}$ are linearly dependent, which implies that the spaces $E^{-}(\lambda)$ and $E^{+}(\lambda)$ have a nontrivial intersection, and this intersection contains the eigenfunctions of (3a). Therefore, $D(\lambda)=0$ if and only if $\lambda \in \sigma_{\mathrm{pt}}(\mathcal{L})$.

Let $\mathcal{C}$ denote the connected component of $\mathbf{C} \backslash \sigma_{\text {ess }}(\mathcal{L})$ containing the right halfplane. We can choose the basis vectors $y_{i}$ to be analytic functions of $\lambda$ in the region $\mathcal{C}$. The Evans function will then also be analytic in $\mathcal{C}$, and the order of its zeros corresponds to the multiplicity of the eigenvalues of $\mathcal{L}$.

More details on the Evans function and the stability of travelling waves can be found in the landmark paper by Alexander, Gardner and Jones 2] and the review article by Sandstede [33].

2.1. The Evans function near infinity. We are interested in the behaviour of $D(\lambda)$ and numerical approximations to $D(\lambda)$ as $|\lambda| \rightarrow \infty$, because experiments show an unexpected deterioration of the approximations in this limit 3 . For simplicity, we will restrict ourselves to scalar reaction-diffusion equations, i.e., we assume that $n=1$. However, it is expected that the methods of analysis presented in this paper also apply to the nonscalar case, though the computations will obviously be more involved.

We may assume without loss of generality that the diffusion coefficient is 1 , so that the partial differential equation reads

$$
u_{t}=u_{x x}+f(u) .
$$

The corresponding eigenvalue problem (3) in this case is

$$
\frac{\mathrm{d} y}{\mathrm{~d} \xi}=A(\xi ; \lambda) y,
$$

where

$$
A(\xi ; \lambda)=\left[\begin{array}{cc}
0 & 1 \\
\lambda-f^{\prime}(\hat{u}(\xi)) & -c
\end{array}\right] .
$$


The limits of $A$ as $\xi \rightarrow \pm \infty$ are given by

$$
A_{ \pm}(\lambda)=\left[\begin{array}{cc}
0 & 1 \\
\lambda-f^{\prime}\left(\hat{u}_{ \pm}\right) & -c
\end{array}\right] .
$$

Furthermore, the eigenvalues of $A_{-}(\lambda)$ are

$$
\mu_{-}^{[1]}, \mu_{-}^{[2]}=\frac{1}{2}\left(-c \pm \sqrt{c^{2}+4\left(\lambda-f^{\prime}\left(\hat{u}_{-}\right)\right)}\right) .
$$

To avoid any confusion between the eigenvalues of the differential operator $\mathcal{L}$, which form the point spectrum that we want to compute, and the eigenvalues of the matrices $A_{ \pm}(\lambda)$, we call the latter spatial eigenvalues.

One of the spatial eigenvalues is purely imaginary if $\lambda$ lies on the parabolic curve given by

$$
\gamma_{-}=\left\{-s^{2}+f^{\prime}\left(\hat{u}_{-}\right)+1 c s: s \in \mathbf{R}\right\} .
$$

The curve $\gamma_{-}$is part of the essential spectrum. If $\lambda$ lies to the right of $\gamma_{-}$, then the spatial eigenvalues $\mu_{-}^{[1]}$ and $\mu_{-}^{[2]}$ have positive and negative real parts, respectively.

The limit $\xi \rightarrow+\infty$ is treated in the same manner and leads to the curve $\gamma_{+}$. The region $\mathcal{C}$ on which the Evans function is defined is the part of the complex plane to the right of $\gamma_{-} \cup \gamma_{+}$.

We assume henceforth that $\lambda \in \mathcal{C}$. We compute the asymptotic behaviour of the Evans function as $|\lambda| \rightarrow \infty$ using a different approach to that outlined in Alexander, Gardner and Jones [2, $\S 5 \mathrm{~B}$ ], extending the approximation to further higher order corrections. We start with the solution $y$ of (4) satisfying $y(\xi) \rightarrow 0$ as $\xi \rightarrow-\infty$. The matrix $A$ in (4b) goes to $A_{-}$as defined in (5) in this limit, and the eigenvalues of $A_{-}$are given in (6), with corresponding eigenvectors $\left(1, \mu_{-}^{[1]}\right)^{\top}$ and $\left(1, \mu_{-}^{[2]}\right)^{\top}$. This suggests writing $y$ as

$$
y(\xi)=\exp \left(\mu_{-}^{[1]} \xi\right)\left(\bar{u}(\xi)\left[\begin{array}{c}
1 \\
\mu_{-}^{[1]}
\end{array}\right]+\bar{v}(\xi)\left[\begin{array}{c}
1 \\
\mu_{-}^{[2]}
\end{array}\right]\right)=\exp \left(\mu_{-}^{[1]} \xi\right) B \bar{y}(\xi)
$$

where

$$
\bar{y}=\left[\begin{array}{l}
\bar{u} \\
\bar{v}
\end{array}\right] \text { and } B=\left[\begin{array}{cc}
1 & 1 \\
\mu_{-}^{[1]} & \mu_{-}^{[2]}
\end{array}\right] .
$$

The vector $\bar{y}$ satisfies the linear differential equation

$$
\frac{\mathrm{d} \bar{y}}{\mathrm{~d} \xi}=\bar{A}(\xi ; \lambda) \bar{y} \quad \text { with } \quad \bar{A}=\left(B^{-1} A B-\mu_{-}^{[1]} I\right) .
$$

The matrix $\bar{A}(\xi ; \lambda)$ in this equation is given by

$$
\bar{A}(\xi ; \lambda)=\left[\begin{array}{cc}
-\frac{1}{\kappa} \varphi_{-}(\xi) & -\frac{1}{\kappa} \varphi_{-}(\xi) \\
\frac{1}{\kappa} \varphi_{-}(\xi) & -\kappa+\frac{1}{\kappa} \varphi_{-}(\xi)
\end{array}\right],
$$

where

$$
\varphi_{-}(\xi)=f^{\prime}(\hat{u}(\xi))-f^{\prime}\left(\hat{u}_{-}\right) \text {and } \kappa=\sqrt{c^{2}+4\left(\lambda-f^{\prime}\left(\hat{u}_{-}\right)\right)} .
$$

Note that the parameters $c$ and $\lambda$ are replaced by only one parameter, $\kappa$. Now, suppose that $\bar{u}$ and $\bar{v}$ can be expanded in inverse powers of $\kappa$ :

$$
\begin{aligned}
& \bar{u}(\xi ; \kappa)=\bar{u}_{0}(\xi)+\kappa^{-1} \bar{u}_{1}(\xi)+\kappa^{-2} \bar{u}_{2}(\xi)+\kappa^{-3} \bar{u}_{3}(\xi)+\mathcal{O}\left(\kappa^{-4}\right), \\
& \bar{v}(\xi ; \kappa)=\bar{v}_{0}(\xi)+\kappa^{-1} \bar{v}_{1}(\xi)+\kappa^{-2} \bar{v}_{2}(\xi)+\kappa^{-3} \bar{v}_{3}(\xi)+\mathcal{O}\left(\kappa^{-4}\right) .
\end{aligned}
$$


If we substitute these expansions in (8) and equate the coefficients of the powers of $\kappa$, we find:

$$
\begin{aligned}
0 & =0, & 0 & =-\bar{v}_{0}, \\
\bar{u}_{0}^{\prime} & =0, & \bar{v}_{0}^{\prime} & =-\bar{v}_{1}, \\
\bar{u}_{1}^{\prime} & =-\varphi_{-}(\xi)\left(\bar{u}_{0}+\bar{v}_{0}\right), & \bar{v}_{1}^{\prime} & =\varphi_{-}(\xi)\left(\bar{u}_{0}+\bar{v}_{0}\right)-\bar{v}_{2}, \\
\bar{u}_{2}^{\prime} & =-\varphi_{-}(\xi)\left(\bar{u}_{1}+\bar{v}_{1}\right), & \bar{v}_{2}^{\prime} & =\varphi_{-}(\xi)\left(\bar{u}_{1}+\bar{v}_{1}\right)-\bar{v}_{3} .
\end{aligned}
$$

Assuming that $\bar{u}(\xi)$ and $\bar{v}(\xi)$ are bounded as $\xi \rightarrow-\infty$, the solution of these equations (up to a multiplicative constant) is

$$
\begin{aligned}
& \bar{u}(\xi ; \kappa)=1-\kappa^{-1} \Phi_{-}(\xi)+\frac{1}{2} \kappa^{-2}\left(\Phi_{-}(\xi)\right)^{2}+\mathcal{O}\left(\kappa^{-3}\right), \\
& \bar{v}(\xi ; \kappa)=\kappa^{-2} \varphi_{-}(\xi)+\mathcal{O}\left(\kappa^{-3}\right),
\end{aligned}
$$

where

$$
\Phi_{-}(\xi)=\int_{-\infty}^{\xi} \varphi_{-}(x) \mathrm{d} x
$$

We can do something similar to find the solution $y$ of (4) satisfying $y(\xi) \rightarrow 0$ as $\xi \rightarrow+\infty$. Instead of (7), we write $y$ as

$$
y(\xi)=\exp \left(\mu_{+}^{[2]} \xi\right) B_{+} \bar{y}_{+}(\xi) \quad \text { where } \quad B_{+}=\left[\begin{array}{cc}
1 & 1 \\
\mu_{+}^{[1]} & \mu_{+}^{[2]}
\end{array}\right] .
$$

Expanding $\bar{y}_{+}$in negative powers of $\kappa_{+}$, where

$$
\kappa_{+}=\sqrt{c^{2}+4\left(\lambda-f^{\prime}\left(\hat{u}_{+}\right)\right)}
$$

similar to (9b), we find that

$$
\bar{y}_{+}=\left[\begin{array}{l}
\bar{u}_{+} \\
\bar{v}_{+}
\end{array}\right] \text {with }\left\{\begin{array}{l}
\bar{u}_{+}(\xi ; \kappa)=\kappa_{+}^{-2} \varphi_{+}(\xi)+\mathcal{O}\left(\kappa_{+}^{-3}\right), \\
\bar{v}_{+}(\xi ; \kappa)=1+\kappa_{+}^{-1} \Phi_{+}(\xi)+\frac{1}{2} \kappa_{+}^{-2}\left(\Phi_{+}(\xi)\right)^{2}+\mathcal{O}\left(\kappa_{+}^{-3}\right),
\end{array}\right.
$$

where

$$
\varphi_{+}(\xi)=f^{\prime}(\hat{u}(\xi))-f^{\prime}\left(\hat{u}_{+}\right) \quad \text { and } \quad \Phi_{+}(\xi)=\int_{\xi}^{\infty} \varphi_{+}(x) \mathrm{d} x .
$$

The Evans function is obtained by evaluating both the solution satisfying $y(\xi) \rightarrow 0$ as $\xi \rightarrow-\infty$ and the one satisfying $y(\xi) \rightarrow 0$ as $\xi \rightarrow+\infty$ at $\xi=0$, collecting the resulting vectors in a matrix and computing the determinant of this matrix. This yields

$$
\begin{aligned}
D(\lambda)= & (B \bar{y}(0)) \wedge\left(B_{+} \bar{y}_{+}(0)\right) \\
= & {\left[\begin{array}{cc}
1 & 1 \\
\frac{1}{2}(\kappa-c) & -\frac{1}{2}(\kappa+c)
\end{array}\right]\left[\begin{array}{l}
\bar{u}(0) \\
\bar{v}(0)
\end{array}\right] \wedge\left[\begin{array}{cc}
1 & 1 \\
\frac{1}{2}\left(\kappa_{+}-c\right) & -\frac{1}{2}\left(\kappa_{+}+c\right)
\end{array}\right]\left[\begin{array}{l}
\bar{u}_{+}(0) \\
\bar{v}_{+}(0)
\end{array}\right] } \\
= & \frac{1}{2}\left(\kappa-\kappa_{+}\right)\left(\bar{v}(0) \bar{v}_{+}(0)-\bar{u}(0) \bar{u}_{+}(0)\right) \\
& \quad+\frac{1}{2}\left(\kappa+\kappa_{+}\right)\left(\bar{v}(0) \bar{u}_{+}(0)-\bar{u}(0) \bar{v}_{+}(0)\right) .
\end{aligned}
$$

Substituting (10) and (12) and using the fact that $\kappa-\kappa_{+}=\mathcal{O}\left(|\lambda|^{-1 / 2}\right)$ as $|\lambda| \rightarrow \infty$, we find that

$$
\begin{aligned}
D(\lambda) & =-\frac{1}{2}\left(\kappa+\kappa_{+}\right) \bar{u}(0) \bar{v}_{+}(0)+\mathcal{O}\left(\kappa^{-2}\right) \\
& =-2 \lambda^{1 / 2}+\Phi-\frac{1}{4} \lambda^{-1 / 2}\left(\Phi^{2}-2 f^{\prime}\left(\hat{u}_{-}\right)-2 f^{\prime}\left(\hat{u}_{+}\right)+c^{2}\right)+\mathcal{O}\left(\lambda^{-1}\right),
\end{aligned}
$$


where

$$
\Phi=\Phi_{-}(0)+\Phi_{+}(0)=\int_{-\infty}^{0} \varphi_{-}(x) \mathrm{d} x+\int_{0}^{\infty} \varphi_{+}(x) \mathrm{d} x .
$$

The approach of Sandstede 33 yields $D(\lambda)=-2 \lambda^{1 / 2}+\mathcal{O}(1)$. This agrees with (14), but the approach presented here gives two more terms. Furthermore, we can easily find additional terms by extending the expansions (10).

\section{Magnus methods}

If we want to evaluate the Evans function numerically, we have to solve the differential equation (3). Moan [25] studied methods based on the Magnus series for the solution of Sturm-Liouville problems of the form $-\left(p y^{\prime}\right)^{\prime}+q y=\lambda w y$ on a finite interval. Moan noticed that some of the quantities involved in the computation are independent on the spectral parameter $\lambda$ and hence need to be computed only once when solving the differential equation for several values of $\lambda$. Moan also proposed a modification of the method based on summing some of the terms analytically which improves the accuracy when $\lambda$ is large.

Jódar and Marletta 21 noticed that the Magnus method in combination with the compound matrix method performs well on some scalar Sturm-Liouville problems of high order; see also Greenberg and Marletta [14.

This approach was generalized by Aparicio, Malham and Oliver [3], who proposed to use a Magnus method for solving the boundary value problem (3). They mentioned the robustness across all regimes as an advantage of Magnus integrators. Furthermore, they pointed out that the computational cost of Magnus methods, as well as other methods, can be decreased by means of a precomputation technique. In this section, we further analyze the behaviour of the Magnus method in the regime where $\lambda$ is large in modulus.

Magnus 24] showed that the solution of the differential equation $y^{\prime}=A(\xi) y$ can be written as $y(\xi)=\exp (\Omega(\xi)) y(0)$, where the matrix $\Omega(\xi)$ is given by the infinite series

$$
\begin{aligned}
\Omega(\xi)=\int_{0}^{\xi} & A(x) \mathrm{d} x-\frac{1}{2} \int_{0}^{\xi}\left[\int_{0}^{x_{1}} A\left(x_{2}\right) \mathrm{d} x_{2}, A\left(x_{1}\right)\right] \mathrm{d} x_{1} \\
& +\frac{1}{12} \int_{0}^{\xi}\left[\int_{0}^{x_{1}} A\left(x_{2}\right) \mathrm{d} x_{2},\left[\int_{0}^{x_{1}} A\left(x_{2}\right) \mathrm{d} x_{2}, A\left(x_{1}\right)\right]\right] \mathrm{d} x_{1} \\
& +\frac{1}{4} \int_{0}^{\xi}\left[\int_{0}^{x_{1}}\left[\int_{0}^{x_{2}} A\left(x_{3}\right) \mathrm{d} x_{3}, A\left(x_{2}\right)\right] \mathrm{d} x_{2}, A\left(x_{1}\right)\right] \mathrm{d} x_{1}+\cdots
\end{aligned}
$$

where $[\cdot, \cdot]$ denotes the matrix commutator defined by $[X, Y]=X Y-Y X$. Moan and Niesen [26] proved that the series converges if $\int_{0}^{\xi}\|A(x)\| \mathrm{d} x<\pi$.

The Magnus series can be used to solve linear differential equations numerically, if we truncate the infinite series and approximate the integrals numerically. For instance, if we retain only the first term in the series and approximate $A(x)$ by the value at the midpoint, we get $\Omega(\xi) \approx h A\left(\frac{1}{2} \xi\right)$. The resulting one-step method is defined by

$$
y_{k+1}=\exp \left(h A\left(\xi_{k}+\frac{1}{2} h\right)\right) y_{k},
$$

where $h$ denotes the step size and $y_{k}$ approximates the solution at $\xi_{k}=\xi_{0}+k h$. This method is called the Lie midpoint or exponential midpoint method. It is a 
second-order method: the difference between the numerical and the exact solution at a fixed point $\xi$ is $\mathcal{O}\left(h^{2}\right)$.

We can get a fourth-order method by truncating the Magnus series (15) after the second term. We replace the matrix $A(\xi)$ by the linear function $A_{0}+\xi A_{1}$ which agrees with $A(\xi)$ at the two Gauss-Legendre points

$$
\xi_{k}^{[1]}=\xi_{k}+\left(\frac{1}{2}-\frac{1}{6} \sqrt{3}\right) h \quad \text { and } \quad \xi_{k}^{[2]}=\xi_{k}+\left(\frac{1}{2}+\frac{1}{6} \sqrt{3}\right) h .
$$

This yields the scheme

$$
y_{k+1}=\exp \left(\Omega_{k}\right) y_{k}
$$

where

$$
\Omega_{k}=\frac{1}{2} h\left(A\left(\xi_{k}^{[1]}\right)+A\left(\xi_{k}^{[2]}\right)\right)-\frac{\sqrt{3}}{12} h^{2}\left[A\left(\xi_{k}^{[1]}\right), A\left(\xi_{k}^{[2]}\right)\right] .
$$

The reader is referred to the review paper by Iserles, Munthe-Kaas, Nørsett and Zanna 20] for more information on Magnus and related methods.

If we define $\bar{y}_{k}$ by $y_{k}=\exp \left(\mu_{-}^{[1]} \xi\right) B \bar{y}_{k}$, as suggested by (7), then the fourth-order method (18) transforms to

$$
\bar{y}_{k+1}=\exp \left(\bar{\Omega}_{k}\right) \bar{y}_{k},
$$

where

$$
\bar{\Omega}_{k}=\frac{1}{2} h\left(\bar{A}\left(\xi_{k}^{[1]}\right)+\bar{A}\left(\xi_{k}^{[2]}\right)\right)-\frac{\sqrt{3}}{12} h^{2}\left[\bar{A}\left(\xi_{k}^{[1]}\right), \bar{A}\left(\xi_{k}^{[2]}\right)\right],
$$

with $\bar{A}$ as given in (9). So applying the Magnus method to the transformed equation (8) and transforming the result back to the original coordinate system is the same as applying it to the original equation. This can be explained by the equivariance of the Magnus method under linear transformations and exponential rescalings 9 .

Substitution of (9) in (19b) yields

$$
\bar{\Omega}_{k}=h\left[\begin{array}{cc}
-\kappa^{-1} \alpha_{k} & \beta_{k}-\kappa^{-1} \alpha_{k} \\
\beta_{k}+\kappa^{-1} \alpha_{k} & -\kappa+\kappa^{-1} \alpha_{k}
\end{array}\right]
$$

with

$$
\alpha_{k}=\frac{1}{2}\left(\varphi_{-}\left(\xi_{k}^{[1]}\right)+\varphi_{-}\left(\xi_{k}^{[2]}\right)\right) \quad \text { and } \quad \beta_{k}=-\frac{\sqrt{3}}{12} h\left(\varphi_{-}\left(\xi_{k}^{[1]}\right)-\varphi_{-}\left(\xi_{k}^{[2]}\right)\right) .
$$

Note that $\alpha_{k}$ and $\beta_{k}$ approximate $\varphi_{-}$and $\frac{1}{12} h^{2} \varphi_{-}^{\prime}$ respectively. Furthermore, the exponential midpoint rule (16) is also given by (20a), but with $\alpha_{k}=\varphi_{-}\left(\xi_{k}+\frac{1}{2} h\right)$ and $\beta_{k}=0$ instead of (20b).

3.1. Estimates for the local error. The local error of a one-step method is the difference between the numerical solution and the exact solution after one step. For the Magnus method, the local error is

$$
L_{k}=\exp \left(\Omega_{k}\right) y\left(\xi_{k}\right)-y\left(\xi_{k+1}\right)
$$

or, in transformed coordinates,

$$
\bar{L}_{k}=\exp \left(\bar{\Omega}_{k}\right) \bar{y}\left(\xi_{k}\right)-\bar{y}\left(\xi_{k+1}\right) .
$$

The exponential of the matrix $\bar{\Omega}_{k}$ is most easily calculated by diagonalization: if $\bar{\Omega}_{k}=V_{k} \Lambda_{k} V_{k}^{-1}$ with $\Lambda_{k}$ diagonal, then $\exp \left(\bar{\Omega}_{k}\right)=V_{k} \exp \left(\Lambda_{k}\right) V_{k}^{-1}$ and $\exp \left(\Lambda_{k}\right)$ is 
formed by simply exponentiating the entries on the diagonal. In fact, the diagonal entries of $\Lambda_{k}$ are

$$
\begin{aligned}
& \lambda_{k}^{[1]}=h\left(\kappa^{-1}\left(\beta_{k}^{2}-\alpha_{k}\right)-\kappa^{-3}\left(\beta_{k}^{2}-\alpha_{k}\right)^{2}+\mathcal{O}\left(\kappa^{-5}\right)\right), \\
& \lambda_{k}^{[2]}=-h\left(\kappa+\kappa^{-1}\left(\beta_{k}^{2}-\alpha_{k}\right)-\kappa^{-3}\left(\beta_{k}^{2}-\alpha_{k}\right)^{2}+\mathcal{O}\left(\kappa^{-5}\right)\right),
\end{aligned}
$$

as $|\kappa| \rightarrow \infty$. The definition of $\kappa$ in (9b) implies that $\operatorname{Re} \kappa>0$ unless $\lambda$ is real and $\lambda \leq f^{\prime}\left(\hat{u}_{-}\right)-\frac{1}{4} c^{2}$. Under this condition, $-\operatorname{Re} \lambda_{k}^{[2]} \gg 1$ if $h|\kappa| \gg 1$ and hence $\exp \left(\lambda_{k}^{[2]}\right)$ is exponentially small.

We now assume that we are in the regime with $|\lambda| \gg h^{-2}, h \rightarrow 0$, and $\lambda$ bounded away from the negative real axis in the sense that $|\arg \lambda|\langle\pi-\varepsilon$ where $\varepsilon>0$. In this regime, $\exp \left(\lambda_{k}^{[2]}\right)$ is exponentially small. Taking this into account, a lengthy but straightforward calculation shows that

$$
\exp \left(\bar{\Omega}_{k}\right)=\left[\begin{array}{cc}
1-\frac{h \chi_{k}}{\kappa}+\mathcal{O}\left(\kappa^{-2}\right) & \frac{\beta_{k}}{\kappa}-\frac{\alpha_{k}+h \beta_{k} \chi_{k}}{\kappa^{2}}+\mathcal{O}\left(\kappa^{-3}\right) \\
\frac{\beta_{k}}{\kappa}+\frac{\alpha_{k}+h \beta_{k} \chi_{k}}{\kappa^{2}}+\mathcal{O}\left(\kappa^{-3}\right) & \frac{\beta_{k}^{2}}{\kappa^{2}}-\frac{h \beta_{k}^{2} \chi_{k}}{\kappa^{3}}+\mathcal{O}\left(\kappa^{-4}\right)
\end{array}\right]
$$

where $\chi_{k}=\alpha_{k}-\beta_{k}^{2}$. Substituting this result and the approximation (10) for the exact solution in the definition (21), and using the definitions of $\chi_{k}, \beta_{k}$, and $\Phi_{-}$, we find that the local error of the Magnus method is given by

$$
\bar{L}_{k}=\left[\begin{array}{c}
\kappa^{-1} \gamma_{k}+\mathcal{O}\left(\kappa^{-2} h^{4}\right) \\
\kappa^{-1} \beta_{k}+\mathcal{O}\left(\kappa^{-2} h\right)
\end{array}\right]
$$

where

$$
\begin{aligned}
\gamma_{k} & =\int_{\xi_{k}}^{\xi_{k+1}} \varphi_{-}(x) \mathrm{d} x-h\left(\alpha_{k}-\beta_{k}^{2}\right) \\
& =h^{5}\left(\frac{1}{4320} \varphi^{\prime \prime \prime \prime}\left(\xi_{k}+\frac{1}{2} h\right)+\frac{1}{144}\left(\varphi^{\prime}\left(\xi_{k}+\frac{1}{2} h\right)\right)^{2}\right)+\mathcal{O}\left(h^{7}\right)
\end{aligned}
$$

and

$$
\beta_{k}=\frac{1}{12} h^{2} \varphi^{\prime}\left(\xi_{k}+\frac{1}{2} h\right)+\mathcal{O}\left(h^{4}\right) .
$$

In deriving the above expression, we also replaced $\varphi_{-}(\xi)$ by $\varphi(\xi)=f^{\prime}(\hat{u}(\xi))$. This is allowed since they differ by a constant term, and only the derivative appears in (24).

Equation (24) gives the local error of the fourth-order Magnus method (18) in transformed variables. Since the method has order four, the local error is $\mathcal{O}\left(h^{5}\right)$ as $h \rightarrow 0$ when solving a fixed equation. However, in our case, the constraint $|\lambda| \gg h^{-2}$ implies that $\lambda$, and hence the relative influence of the coefficients in the equation, must change as $h$ approaches zero. It turns out that the local error is $\mathcal{O}\left(h^{2}\right)$ in this setting. In other words, the method behaves like a first-order method (globally). This phenomenon is called order reduction.

The cause of this order reduction is the stiffness of the differential equation (44). Indeed, if we define the stiffness ratio as the quotient between the largest and smallest eigenvalue (as in Iserles [18]), then the stiffness quotient is

$$
\left|\frac{\lambda_{k}^{[2]}}{\lambda_{k}^{[1]}}\right|=\left|\frac{\kappa^{2}}{\beta_{k}^{2}-\alpha_{k}}\right|+\mathcal{O}(1),
$$


where the leading term shown grows like $|\lambda|$. Hence, the problem is stiff if $\lambda$ is large in modulus, causing troubles for the numerical method.

3.2. Estimates for the global error. The global error is the error of the numerical method after several steps, say $k$. Hence, the global error is $E_{k}=y_{k}-y\left(\xi_{k}\right)$, with $y_{k}$ defined by the numerical method starting from $y_{0}=y\left(\xi_{0}\right)$. For the Magnus method (18), the global error satisfies the recursion relation $E_{k+1}=\exp \left(\Omega_{k}\right) E_{k}+L_{k}$ with $E_{0}=0$, or, in transformed coordinates,

$$
\bar{E}_{k+1}=\exp \left(\bar{\Omega}_{k}\right) \bar{E}_{k}+\bar{L}_{k}, \quad \bar{E}_{0}=0 .
$$

A routine induction argument using (23) and (24) shows that the leading term of the global error is given by

$$
\bar{E}_{k}=\left[\begin{array}{c}
\kappa^{-1} \sum_{j=0}^{k-1} \gamma_{j}+\mathcal{O}\left(\kappa^{-2} h^{4}\right) \\
\kappa^{-1} \beta_{k-1}+\mathcal{O}\left(\kappa^{-2} h\right)
\end{array}\right] .
$$

This shows an advantage of stiffness: the exact flow quickly reduces the error in the stiff component. The Magnus method inherits this property here and annihilates at every step the error in the stiff component up to leading order (if $|\kappa| h \gg 1$ ). On the other hand, the first (nonstiff) component of the error is propagated without change. Since the local error in the stiff component is much bigger than the error in the nonstiff component (order $h^{2}$ versus order $h^{5}$ ), we arrive at the surprising conclusion that the local and global error are equal at leading order.

Substituting the definitions of $\alpha_{k}, \beta_{k}$, and $\gamma_{k}$ in (20b) and (25) and approximating the sum by an integral, we find that

$$
\bar{E}_{k}=\left[\begin{array}{c}
\kappa^{-1} h^{4}\left(\frac{1}{4320}\left(\varphi^{\prime \prime \prime}\left(\xi_{k}\right)-\varphi^{\prime \prime \prime}\left(\xi_{0}\right)\right)+\frac{1}{144} \int_{\xi_{0}}^{\xi_{k}}\left(\varphi^{\prime}(\xi)\right)^{2} \mathrm{~d} \xi\right)+\mathcal{O}\left(\kappa^{-1} h^{6}, \kappa^{-2} h^{4}\right) \\
\frac{1}{12} \kappa^{-1} h^{2} \varphi^{\prime}\left(\xi_{k}-\frac{1}{2} h\right)+\mathcal{O}\left(\kappa^{-1} h^{4}, \kappa^{-2} h\right)
\end{array}\right] .
$$

We see that the global error is $\mathcal{O}\left(h^{2}\right)$. Usually, a factor $h$ is lost in the transition from the local to the global error, but here both the local and the global error are $\mathcal{O}\left(h^{2}\right)$, because the local error is mainly in the stiff component. Hence, the fourth-order Magnus method given in (18) behaves like a second-order method if one considers the global error. The numerical experiments in Section 5 support this analysis. However, the fact that the global error is $\mathcal{O}\left(h^{2}\right)$ does not tell the whole story, as the relative error (the error divided by the magnitude of the solution) may give a better picture. Indeed, it follows from (77) and (10) that the solution $y$ grows as $\sqrt{\lambda}$, so the relative error is $\mathcal{O}\left(h^{2} / \sqrt{\lambda}\right)$. In other words, the relative error decreases as $|\lambda| \rightarrow \infty$.

We can compute the error associated with the solution satisfying the boundary condition that $y(\xi) \rightarrow 0$ as $\xi \rightarrow+\infty$ similarly. Instead of (28), we now have

$$
\bar{E}_{k}^{+}=\left[\begin{array}{c}
\kappa_{+}^{-1} \sum_{j=0}^{k-1} \gamma_{j}^{+}+\mathcal{O}\left(\kappa_{+}^{-2} h^{4}\right) \\
\kappa_{+}^{-1} \beta_{k-1}^{+}+\mathcal{O}\left(\kappa_{+}^{-2} h\right)
\end{array}\right]
$$

where $\alpha_{k}^{+}, \beta_{k}^{+}$and $\gamma_{k}^{+}$are as given in (20b), with $\varphi_{+}$and $\Phi_{+}$replacing $\varphi_{-}$and $\Phi_{-}$, respectively, and $\kappa_{+}$is as given in (11). 
3.3. The error in the Evans function. The Evans function given in (13) is

$$
D(\lambda)=(B \bar{y}(0)) \wedge\left(B_{+} \bar{y}_{+}(0)\right) .
$$

The numerical error when evaluating the Evans function is therefore

$$
E_{D}=(B \bar{y}(0)) \wedge\left(B_{+} \bar{E}_{k}^{+}\right)+\left(B \bar{E}_{k}\right) \wedge\left(B_{+} \bar{y}_{+}(0)\right)+\left(B \bar{E}_{k}\right) \wedge\left(B_{+} \bar{E}_{k}^{+}\right) .
$$

We can expand this in the same manner as in (13). The first term on the right-hand side becomes

$$
\frac{1}{2}\left(\kappa-\kappa_{+}\right)\left([\bar{E}]_{2} \bar{v}_{+}(0)-[\bar{E}]_{1} \bar{u}_{+}(0)\right)+\frac{1}{2}\left(\kappa+\kappa_{+}\right)\left([\bar{E}]_{2} \bar{u}_{+}(0)-[\bar{E}]_{1} \bar{v}_{+}(0)\right) .
$$

The dominating term in this expression is $-\frac{1}{2}\left(\kappa+\kappa_{+}\right)[\bar{E}]_{1} \bar{v}_{+}(0)$, which is $\mathcal{O}\left(\lambda^{0} h^{4}\right)$; all other terms are $\mathcal{O}\left(\lambda^{-1} h^{2}\right)$ or smaller (recall that we assumed that $|\lambda| \gg h^{-2}$ ). Therefore, the dominating contribution to the error in the Evans function comes from the first (nonstiff) component of the global error, even though the second (stiff) component is larger. This is because the stiff and nonstiff directions are exchanged when you integrate in the other direction. Hence, when taking the wedge product of the global error of the solution on $[-\infty, 0]$ with the solution itself on $[0,+\infty]$, the stiff component of the global error is paired with the stiff component of the solution; similarly, the nonstiff component of the global error is paired with the nonstiff component of the solution. Since the solution is mainly along the nonstiff direction, the nonstiff component of the global error (which has order $h^{4}$ ) is brought to the forefront, and the stiff component of the global error (which has order $h^{2}$ ) is reduced.

Substituting the exact solution from (10) and (12) and the global error from (28) and (30) in (31), we find that the error in the Evans function is

$$
E_{D}=\sum_{j=0}^{k-1} \gamma_{j}+\sum_{j=0}^{k-1} \gamma_{j}^{+}+\mathcal{O}\left(\lambda^{-1 / 2} h^{4}\right) .
$$

Assuming that the differential equation is solved on the intervals $[-L, 0]$ and $[0, L]$, with $L=N h$, this evaluates to

$$
\begin{array}{r}
E_{D}=\left[h \sum_{j=-N}^{N-1}\left(\varphi\left(j h+\left(\frac{1}{2}-\frac{1}{6} \sqrt{3}\right) h\right)+\varphi\left(j h+\left(\frac{1}{2}+\frac{1}{6} \sqrt{3}\right) h\right)\right)-\int_{-L}^{L} \varphi(x) \mathrm{d} x\right] \\
-h \sum_{j=0}^{k-1} \beta_{j}^{2}-h \sum_{j=0}^{k-1}\left(\beta_{j}^{+}\right)^{2}+\mathcal{O}\left(\lambda^{-1 / 2} h^{4}\right) .
\end{array}
$$

The term within brackets is the difference between the approximation of $\int_{-L}^{L} \varphi(x) \mathrm{d} x$ by two-point Gauss-Legendre quadrature and the integral itself. In our setting, all derivatives of the travelling wave $\hat{u}$, and therefore also of the function $\varphi=f^{\prime} \circ \hat{u}$, vanish at infinity (see 92 ). Now, assuming that $L$ is so large that the derivatives of $\varphi$ at $L$ are negligible, the error in Gauss-Legendre quadrature vanishes at all orders in $h$, for essentially the same reason that the trapezoidal rule is so effective for periodic integrands; this is easily proved with the Euler-MacLaurin formula (see, for instance, Davis and Rabinowitz [7, §3.4]). Hence, only the sums involving 
the $\beta_{j}$ and $\beta_{j}^{+}$survive. These can be approximated easily using (26), and we find that

$$
E_{D}=-\frac{h^{4}}{144} \int_{-\infty}^{\infty}\left(\varphi^{\prime}(x)\right)^{2} \mathrm{~d} x+\mathcal{O}\left(h^{6}\right) \quad \text { with } \quad \varphi(\xi)=f^{\prime}(\hat{u}(\xi)) .
$$

So, in the end, the error in the Evans function is of order $h^{4}$, which is just what one would expect from a fourth-order method.

3.4. The exponential midpoint rule. We saw above that the fourth-order Magnus method (18) suffers severe order reduction when solving (4) with $|\lambda| \gg 1$. This is not the case for all methods. There are even methods based on the Magnus series which do not suffer global order reduction, like the exponential midpoint rule (16) which has order two (this method is also known as the second-order Magnus method). When applied to (4), this method is of the form (19a), (20a) with $\alpha_{k}$ and $\beta_{k}$ given by $\alpha_{k}=\varphi_{-}\left(\xi_{k}+\frac{1}{2} h\right)$ and $\beta_{k}=0$ respectively. If we substitute this in (24), we see that the $\kappa^{-1}$ term in the second (stiff) component drops out, and that the local error is $\left[\mathcal{O}\left(\kappa^{-1} h^{3}\right) \mathcal{O}\left(\kappa^{-2} h\right)\right]^{\top}$. So, the exponential midpoint rule does suffer some local order reduction, but not as severe as the fourth-order Magnus method, for which the second component of the local error is of order $\kappa^{-1} h^{2}$.

The global error can be computed as in $\$ 3.2$. Again, only the nonstiff component propagates, so the global error is $\left[\mathcal{O}\left(\kappa^{-1} h^{2}\right) \mathcal{O}\left(\kappa^{-2} h\right)\right]^{\top}$. As $|\kappa| \gg h^{-1}$, the first component dominates and the exponential midpoint rule effectively does not suffer from order reduction if one looks at the global error.

Continuing to find the error in the Evans function, as we did in $\$ 3.3$ we find

$$
E_{D}=h \sum_{j=-N}^{N-1} \varphi\left(j h+\frac{1}{2} h\right)-\int_{-L}^{L} \varphi(x) \mathrm{d} x+\mathcal{O}\left(\lambda^{-1 / 2} h^{2}\right) .
$$

Comparing with (32) for the fourth-order Magnus method, we see that the sums involving the $\beta_{j}$ and $\beta_{j}^{+}$have dropped out (because $\beta_{j}=0$ ), and that the two-point Gauss-Legendre quadrature is replaced by the trapezoidal rule. Again, the error in the trapezoidal rule vanishes at all orders if $L$ is sufficiently large. Hence, the error in the Evans function is $\mathcal{O}\left(\lambda^{-1 / 2} h^{2}\right)$. In contrast, the fourth-order Magnus method has $E_{D}=\mathcal{O}\left(h^{4}\right)$; see (33). Thus, we can expect the second-order method to be more accurate than the fourth-order method. The experiments in Section 5 confirm this.

\section{The Gauss-Legendre Method}

Most numerical computations of the Evans function reported in the literature use a Runge-Kutta method, in particular the classical explicit fourth-order method and the two-stage Gauss-Legendre method. As the differential equation that we want to solve is stiff, we consider the two-stage Gauss-Legendre method. The method is given by

$$
\begin{aligned}
s_{1} & =A\left(\xi_{k}^{[1]}\right)\left(y_{k}+\frac{1}{4} h s_{1}+\left(\frac{1}{4}-\frac{\sqrt{3}}{6}\right) h s_{2}\right), \\
s_{2} & =A\left(\xi_{k}^{[2]}\right)\left(y_{k}+\left(\frac{1}{4}+\frac{\sqrt{3}}{6}\right) h s_{1}+\frac{1}{4} h s_{2}\right), \\
y_{k+1} & =y_{k}+\frac{1}{2} h\left(s_{1}+s_{2}\right),
\end{aligned}
$$

where $\xi_{k}^{[1]}$ and $\xi_{k}^{[2]}$ are the Gauss-Legendre points, given in (17). 
We will now analyse the error committed by the Gauss-Legendre method when computing the Evans function. After the usual coordinate transformation, cf. (7), and substitution of the matrix given in (9), we can solve the system (35). After a lengthy but relatively straightforward calculation, we find that $\bar{y}_{k+1}=\Psi_{k} \bar{y}_{k}$ with

$$
\Psi_{k}=\left[\begin{array}{cc}
1-\kappa^{-1} h \alpha_{k}+\frac{1}{2} \kappa^{-2} h^{2} \alpha_{k}^{2} & 12 \kappa^{-2} h^{-1} \beta_{k} \\
12 \kappa^{-2} h^{-1} \beta_{k} & 1-12 \kappa^{-1} h^{-1}+72 \kappa^{-2} h^{-2}
\end{array}\right]+\mathcal{O}\left(\kappa^{-3}\right)
$$

where $\alpha_{k}$ and $\beta_{k}$ are as defined in (20b). The local error can now be found by substituting this matrix and the exact solution in $\bar{L}_{k}=\Psi_{k} \bar{y}\left(\xi_{k}\right)-\bar{y}\left(\xi_{k+1}\right)$, cf. (21). This yields

$$
\bar{L}_{k}=\left[\begin{array}{c}
\kappa^{-1} \bar{L}_{k}^{a}+\kappa^{-2} \bar{L}_{k}^{b}+\mathcal{O}\left(\kappa^{-3} h^{5}\right) \\
\kappa^{-2} \bar{L}_{k}^{c}+\mathcal{O}\left(\kappa^{-3} h^{2}\right)
\end{array}\right]
$$

where

$$
\begin{aligned}
\bar{L}_{k}^{a} & =\int_{\xi_{k}}^{\xi_{k}+h} \varphi_{-}(x) \mathrm{d} x-h \alpha_{k}=\mathcal{O}\left(h^{5}\right) \\
\bar{L}_{k}^{b} & =\Phi_{-}\left(\xi_{k}\right)\left(h \alpha_{k}-\int_{\xi_{k}}^{\xi_{k}+h} \varphi_{-}(x) \mathrm{d} x\right)+\frac{1}{2}\left(h \alpha_{k}\right)^{2}-\frac{1}{2}\left(\int_{\xi_{k}}^{\xi_{k}+h} \varphi_{-}(x) \mathrm{d} x\right)^{2} \\
& =-\bar{L}_{k}^{a}\left(\Phi_{-}\left(\xi_{k}\right)+h \alpha_{k}+\frac{1}{2} \bar{L}_{k}^{a}\right)=\mathcal{O}\left(h^{5}\right) \\
\bar{L}_{k}^{c} & =12 h^{-1} \beta_{k}-\varphi_{-}\left(\xi_{k}+h\right)+\varphi_{-}\left(\xi_{k}\right)=\mathcal{O}\left(h^{3}\right)
\end{aligned}
$$

For reasons which will soon become clear, we must retain the $\kappa^{-2}$ term in the above expression, in contrast to (24) for the Magnus method. We see that the two terms in the first (nonstiff) component are of order $\kappa^{-1} h^{5}$ and $\kappa^{-2} h^{5}$, while the second (stiff) component is of order $\kappa^{-2} h^{3}$. This is a similar situation as with the exponential midpoint rule, except that (35) is a fourth-order method.

To find the global error, we solve the recursion relation $\bar{E}_{k+1}=\Psi_{k} \bar{E}_{k}+\bar{L}_{k}$, $\bar{E}_{0}=0$; cf. (27). The solution is

$$
\bar{E}_{k}=\left[\begin{array}{c}
\kappa^{-1} \sum_{j=0}^{k-1} \bar{L}_{j}^{a}+\kappa^{-2} \sum_{j=0}^{k-1}\left(\bar{L}_{j}^{b}-h \alpha_{j} \sum_{i=0}^{j-1} \bar{L}_{i}^{a}\right)+\mathcal{O}\left(\kappa^{-3} h^{4}\right) \\
\kappa^{-2} \sum_{j=0}^{k-1} \bar{L}_{j}^{c}+\mathcal{O}\left(\kappa^{-3} h^{2}\right)
\end{array}\right] .
$$

Again, only the error in the nonstiff component propagates.

Finally, we compute the error in the Evans function. Estimating the various terms in (31), we find that

$$
E_{D}=-\frac{1}{2}\left(\kappa+\kappa_{+}\right)\left([\bar{E}]_{1} \bar{v}_{+}(0)+\bar{u}(0)\left[\bar{E}^{+}\right]_{2}\right)+\mathcal{O}\left(\lambda^{-1 / 2} h^{8}, \lambda^{-3 / 2} h^{2}\right) .
$$

Hence, we wish to compute $\mathcal{X}=[\bar{E}]_{1} \bar{v}_{+}(0)+\bar{u}(0)\left[\bar{E}^{+}\right]_{2}$. Substitution of the exact solution, given in (10) and (12), and the global error (38) yields

$$
\mathcal{X}=\kappa^{-1} \sum_{j=0}^{N}\left(\bar{L}_{j}^{a}+\bar{L}_{j}^{a,+}\right)+\kappa^{-2} \mathcal{X}_{2}+\mathcal{O}\left(\kappa^{-3} h^{4}\right)
$$

where

$$
\mathcal{X}_{2}=\sum_{j=0}^{N}\left(\bar{L}_{j}^{b}-h \alpha_{j} \sum_{i=0}^{j-1} \bar{L}_{i}^{a}-\bar{L}_{j}^{a} \Phi_{+}(0)+\bar{L}_{j}^{b,+}-h \alpha_{j}^{+} \sum_{i=0}^{j-1} \bar{L}_{i}^{a,+}-\bar{L}_{j}^{a,+} \Phi_{-}(0)\right) .
$$


The sum $\sum_{j=0}^{N}\left(\bar{L}_{j}^{a}+\bar{L}_{j}^{a,+}\right)$ is the term within brackets in (32), so again, it vanishes at all orders in $h$. For the $\Phi_{-}$and $\Phi_{+}$terms, we use

$$
\Phi_{-}\left(\xi_{j}\right)=\int_{-\infty}^{\xi_{j}} \varphi_{-}(x) \mathrm{d} x \approx \sum_{i=0}^{j-1} \int_{\xi_{i}}^{\xi_{i}+h} \varphi_{-}(x) \mathrm{d} x=\sum_{i=0}^{j-1}\left(\bar{L}_{i}^{a}+h \alpha_{i}\right),
$$

where the approximate equality becomes exact in the limit $L \rightarrow \infty$; again, we assume that $L$ is so large that we can neglect any errors here. Substitution of (40), its analogue for $\Phi_{+}$, and (37a) and (37b) yields

$$
\begin{aligned}
\mathcal{X}_{2}=-\sum_{j=0}^{N}\left(h \alpha_{j} \bar{L}_{j}^{a}\right. & +h \alpha_{j}^{+} \bar{L}_{j}^{a,+}+\sum_{i=0}^{N}\left(\alpha_{i}^{+} \bar{L}_{j}^{a}+\alpha_{i} \bar{L}_{j}^{a,+}\right) \\
& \left.+\sum_{i=0}^{j-1}\left(h \alpha_{i} \bar{L}_{j}^{a}+h \bar{L}_{i}^{a} \alpha_{j}+h \alpha_{i}^{+} \bar{L}_{j}^{a,+}+h \bar{L}_{i}^{a,+} \alpha_{j}^{+}\right)\right)+\mathcal{O}\left(h^{8}\right),
\end{aligned}
$$

where the remainder term comes from estimating terms like $\sum_{j} \sum_{i} \bar{L}_{i}^{a} \bar{L}_{j}^{a}$. This nested sum can be written as the product of two sums:

$$
\mathcal{X}_{2}=\sum_{i=0}^{N} h\left(\alpha_{i}+\alpha_{i}^{+}\right) \cdot \sum_{j=0}^{N}\left(\bar{L}_{j}^{a}+\bar{L}_{j}^{a,+}\right)+\mathcal{O}\left(h^{8}\right) .
$$

So we arrive again at the sum $\sum_{j=0}^{N}\left(\bar{L}_{j}^{a}+\bar{L}_{j}^{a,+}\right)$, which vanishes at all orders.

Substituting everything back into (39), we find that the error in the Evans function is given by

$$
E_{D}=\mathcal{O}\left(\lambda^{-1 / 2} h^{8}, \lambda^{-1} h^{4}, \lambda^{-3 / 2} h^{2}\right) .
$$

This is clearly better than the fourth-order Magnus method, with $E_{D}=\mathcal{O}\left(\lambda^{0} h^{4}\right)$, and the exponential midpoint rule, with $E_{D}=\mathcal{O}\left(\lambda^{-1 / 2} h^{2}\right)$.

\section{NUMERICAL EXPERIMENTS}

In this section, we evaluate the Evans function for a particular example. The error in this computation is determined and compared against the estimates derived in the previous sections.

The example is the Fisher equation

$$
u_{t}=u_{x x}+u-u^{2} .
$$

This is a reaction-diffusion equation of the form (11). Fisher 12 used it to describe the transmission of genes in a population. It is now viewed as the prototype equation admitting travelling front solutions [28, §11.2].

The Fisher equation supports a travelling wave solution with wave speed $c=$ $-\frac{5}{6} \sqrt{6}$. In fact, this solution is known analytically:

$$
u(x, t)=\hat{u}(\xi)=\frac{1}{\left(1+\mathrm{e}^{\xi / \sqrt{6}}\right)^{2}} \quad \text { where } \quad \xi=x-\frac{5}{6} \sqrt{6} t .
$$

Suppose that we wish to determine the stability of this travelling wave. We are led to consider the eigenvalue problem (4), which in this case reads

$$
\frac{\mathrm{d} y}{\mathrm{~d} \xi}=\left[\begin{array}{cc}
0 & 1 \\
\lambda-\varphi(\xi) & -c
\end{array}\right] y,
$$


where

$$
\varphi(\xi)=1-2 \hat{u}(\xi)=1-\frac{2}{\left(1+\mathrm{e}^{\xi / \sqrt{6}}\right)^{2}} .
$$

We solve this equation with the fourth-order Magnus method, given by (18). In the previous section, we derived the local error estimate (24). For the Fisher equation, this estimate evaluates to

$$
\bar{L}_{k} \approx \frac{1}{\kappa}\left[\begin{array}{c}
\frac{h^{5} \mathrm{e}^{\xi / \sqrt{6}}\left(-8 \mathrm{e}^{3 \xi / \sqrt{6}}+33 \mathrm{e}^{2 \xi / \sqrt{6}}+702 \mathrm{e}^{\xi / \sqrt{6}}+1\right)}{38880\left(1+\mathrm{e}^{\xi / \sqrt{6}}\right)^{6}} \\
\frac{\sqrt{6} h^{2} \mathrm{e}^{\xi / \sqrt{6}}}{18\left(1+\mathrm{e}^{\xi / \sqrt{6}}\right)^{3}}
\end{array}\right]
$$

where $\kappa=\sqrt{c^{2}+4(\lambda+1)}$ and $\xi$ is short for $\xi_{k}$.

The global error estimate is given in (29). Assuming that $\xi_{0}$ is negative and so large in magnitude that we can take $\xi_{0}=-\infty$, we find that

$$
\bar{E}_{k} \approx \frac{1}{\kappa}\left[\begin{array}{c}
\frac{\sqrt{6} h^{4} \mathrm{e}^{\xi / \sqrt{6}}\left(36 \mathrm{e}^{4 \xi / \sqrt{6}}+180 \mathrm{e}^{3 \xi / \sqrt{6}}+364 \mathrm{e}^{2 \xi / \sqrt{6}}+353 \mathrm{e}^{\xi / \sqrt{6}}+1\right)}{38880\left(1+\mathrm{e}^{\xi / \sqrt{6}}\right)^{6}} \\
\frac{\sqrt{6} h^{2} \mathrm{e}^{\xi / \sqrt{6}}}{18\left(1+\mathrm{e}^{\xi / \sqrt{6}}\right)^{3}}
\end{array}\right] .
$$

Finally, estimate (33) for the error in the Evans function is

$$
E_{D} \approx-\frac{\sqrt{6}}{1080} h^{4} \approx-0.002268 h^{4}
$$

This estimate is independent of the parameter $\kappa$.

We perform some numerical experiments to check the validity of these estimates. First, we solve (43) from $\xi=-30$ using the fourth-order Gauss-Legendre method with step size $h=0.02$. We will refer to this solution as the "exact" solution. Then, we take the "exact" solution at $\xi=-1$ and do a single step with the fourth-order Magnus method with step size $h=0.2$ or $h=0.1$. The local error can now be determined by comparing the result of this single step against the "exact" solution; this local error is plotted in the top row of Figure 1, together with the local error estimate (44). The horizontal axis in the plots shows the imaginary part of the eigenvalue parameter $\lambda$, which varies from 1 to $10^{8} 1$ in our experiments.

The global error can be determined by solving (43) from $\xi=-30$ till $\xi=-1$ with the fourth-order Magnus method and comparing it against the "exact" solution. This results in the bottom row of Figure 1. Finally, Figure 2 shows the difference between the Evans function as computed by the Magnus method and the "exact" value, compared against the estimate (46).

All graphs show that the error estimates agree well with the actual error when $\lambda$ is moderately large in magnitude. However, the numerical method starts to break down when $|\lambda|$ increases above $10^{7}$.

The implementation used in the experiments is a straightforward MATLAB code. One detail proved to be important, namely, the computation of the matrix exponential in (18). The standard routine for this is called expm and uses Padé approximation combined with scaling and squaring. However, we found that an alternative approach based on the Schur decomposition and implemented in the 

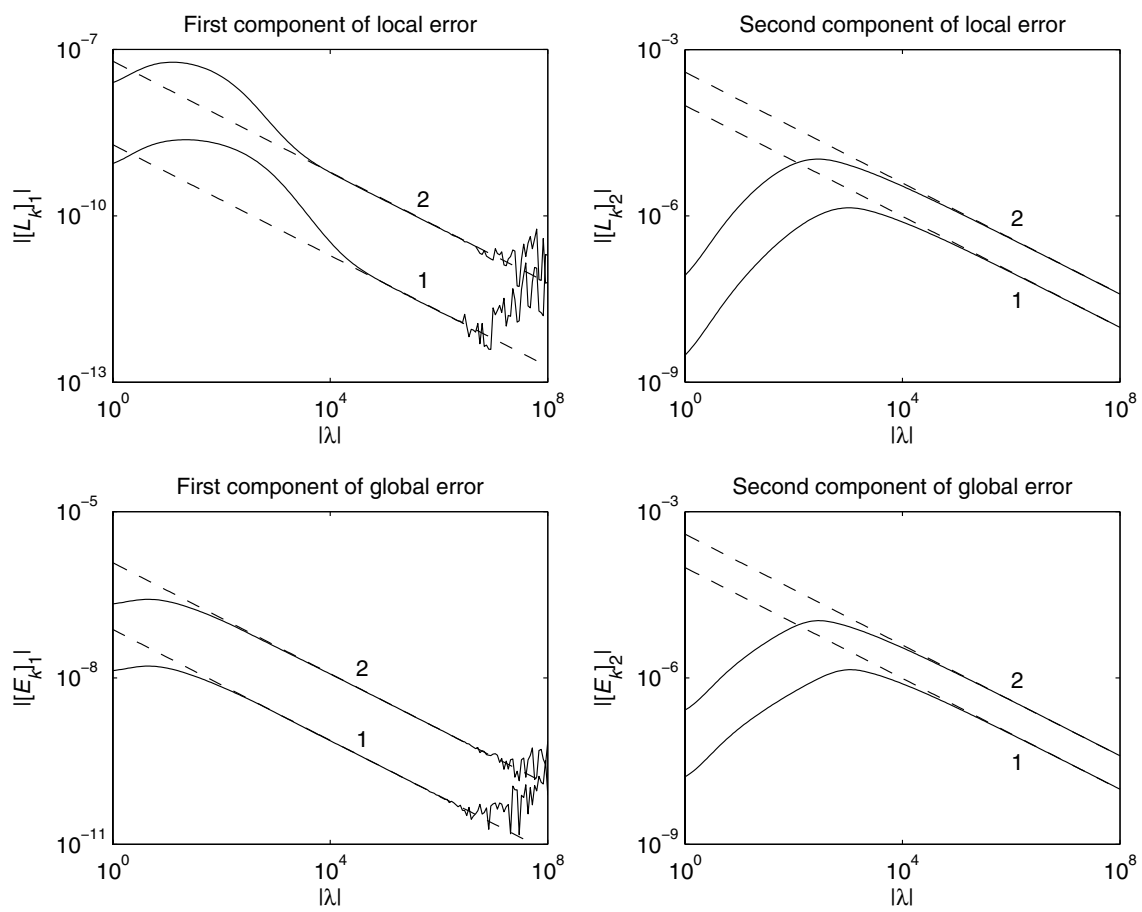

Figure 1. The solid lines in the graphs on the top row show the local error committed by the fourth-order Magnus method. The step size $h$ is 0.1 and 0.2 for the line labelled 1 and 2, respectively. The dash lines show the local error estimate (44). On the bottom row, the solid lines shows the global error and the dash lines show the estimate (45).
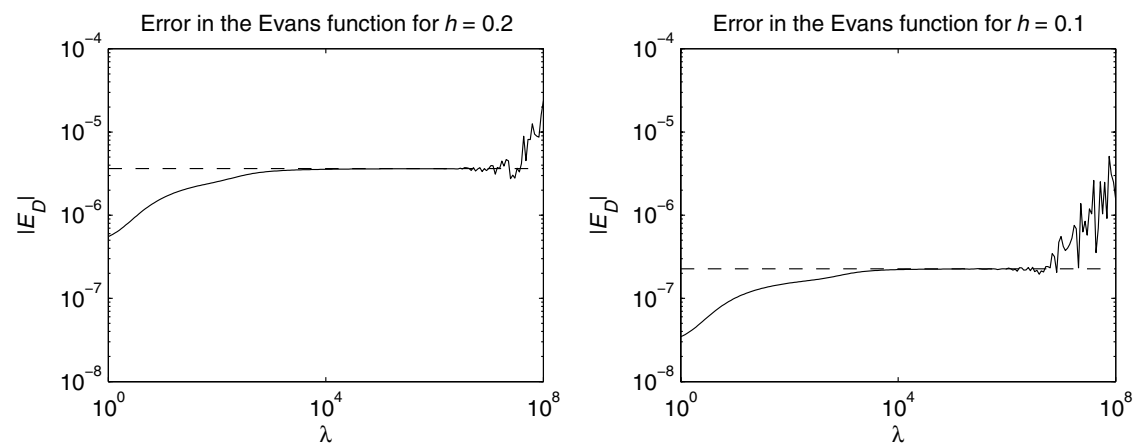

Figure 2. The solid line shows the error in the Evans function as evaluated by the fourth-order Magnus method, while the dash line shows the error estimate (46). The step size is $h=0.2$ for the graph on the left and $h=0.1$ for the graph on the right. 

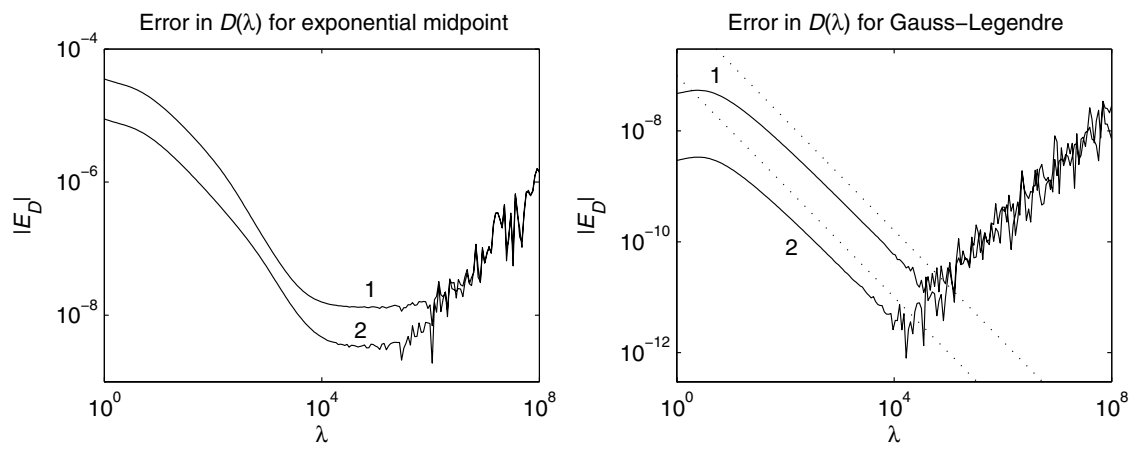

Figure 3. The left graph shows the error in the Evans function as evaluated by the exponential midpoint rule (16), while the right graph shows the same for the fourth-order Gauss-Legendre method (35). The step size is $h=0.2$ for the curve labelled 2 and $h=0.1$ for the curve labelled 1 . The dotted lines in the second plot show $10^{-3} h^{4} /|\lambda|$.

Matlab routine expmdemo3 works better in our case. Specifically, when using Padé approximation, the numerical method loses accuracy around $|\lambda|=10^{5}$, as opposed to $|\lambda|=10^{7}$ for the Schur decomposition. Generally, the Schur decomposition runs into trouble when the matrix to be exponentiated is nearly defective, but in our case the eigenvalues are far apart; cf. (22). The reader is refered to the article by Moler and Van Loan [27] for an extensive discussion on this subject.

When the experiment is repeated with the exponential midpoint rule (16) and the fourth-order Gauss-Legendre method (35), the error in the Evans function is as plotted in Figure 3. We concluded in Section 3.4 that, because the exponential midpoint rule suffers less from order reduction, it is likely to have a smaller error than the fourth-order Magnus method if $|\lambda|$ is large. The error plots confirm this.

For the fourth-order Gauss-Legendre method, which is the method that is more relevant in practice, the error in the Evans function is shown in the right half of Figure 3. We found the estimate (41) for the error, and the numerical results show that the term of order $\lambda^{-1} h^{4}$ dominates: the line $10^{-3}|\lambda|^{-1} h^{4}$ tracks the graphs closely for $|\lambda|$ up to $10^{4}$ (the coefficient $10^{-3}$ was not determined by any computation, in contrast to the coefficient in (46), but it was chosen to give a suitable match). When $|\lambda|>10^{4}$, the error committed by the Gauss-Legendre method starts to increase erratically, following roughly the equation $E_{D}=10^{-12} \sqrt{|\lambda|}$. This is likely due to round-off error, as $|y(\xi)|$ is approximately $\sqrt{|\lambda|}$. This suggests that the loss of accuracy in the fourth-order Magnus method is also due to round-off error, exacerbated by ill-conditioning of the matrix exponential (compare with the influence of the method for computing the matrix exponential, as noted on page 173).

\section{Conclusions}

We found that the fourth-order Magnus method, when applied to the linear differential equation (44) in the regime $|\lambda| \gg 1 / h^{2}$, commits a global error of order $h^{2} / \sqrt{|\lambda|}$ (relative to the exact solution). It is remarkable that the Magnus 
method converges at all. The convergence result 26] for the Magnus series mentioned earlier guarantees convergence only when $|\lambda|<\pi / h^{2}$, so the usual convergence proof for the truncated series does not hold. However, the error analysis in this paper shows that the method does indeed converge for equations of the form (4).

Given that the method converges, it is remarkable that the order of the method drops. This is connected to the concept of stability. The Magnus method solves autonomous linear equations exactly. A fortiori, the numerical solution of Dahlquist's test equation $y^{\prime}=a y$ (with $a \in \mathbf{C}$ ) is stable if and only if the exact solution is stable, meaning that it converges to 0 as $x \rightarrow \infty$. Hence, the Magnus method is A-stable and even L-stable (see, e.g., Hairer and Wanner [16 for a definition of these terms). Nevertheless, the fourth-order Magnus method suffers from order reduction in the current setting, in which the equation is nearly autonomous. This may be connected to the fact that the fourth-order Magnus method is not B-stable. A simple counterexample is given by the equation $y^{\prime}=A y$ with $A(x)=\left[\begin{array}{cc}-1 & 1 \\ 0 & -1\end{array}\right]$ for $x<3$ and $A(x)=\left[\begin{array}{cc}-1 & 0 \\ 1 & -1\end{array}\right]$ for $x>3$. This equation is contractive, but the numerical solution does not preserve contractivity as $h$ increases above 6 . In contrast, the Gauss-Legendre method and the exponential midpoint rule are known to be B-stable, and they do not suffer from order reduction. We again refer to Hairer and Wanner [16] for a precise definition of B-stability and its connection to order reduction.

Similar results were obtained by Hochbruck and Lubich [17, who treated Magnus methods applied to semi-discretized Schrödinger equations. They could prove that the method converges even when there is no known convergence result for the untruncated Magnus series. González, Ostermann and Thalhammer [13 found that the exponential midpoint rule suffers from order reduction when applied to semidiscretized parabolic equations. The matrices in the semi-descretized equations considered by them have negative eigenvalues that are large in magnitude, just as problem (41) treated here.

However, the fourth-order Magnus method regains the full order when combining the solution of the differential equation (44) satisfying the boundary condition at $\xi=-\infty$ with the one satisfying the condition at $\xi=+\infty$ to form the Evans function. As is clearly shown both by the analysis and by the experiment, the error committed by the fourth-order Magnus is of order $h^{4}$ uniformly in $\lambda$. Nevertheless, the Gauss-Legendre method is still superior: its error decreases as $|\lambda|$ increases.

The same holds to a lesser degree for the exponential midpoint rule. The analysis indicates that the error commited by this method is of order $\lambda^{-1 / 2} h^{2}$. The numerical results for the exponential midpoint rule do not quite seem to agree with this, but they also show that the error decreases as a function of $|\lambda|$; the reason for this discrepancy is unknown. Nevertheless, we can conclude that the second term in the Magnus expansion (15) actually harms the numerical algorithm when $\lambda$ is large in magnitude.

This suggests that the Right Correction Magnus Series, as proposed by Degani and Schiff [8], or the modified Magnus method, as proposed by Iserles [19], might perform well on this problem. We ran some preliminary experiments with these methods, which showed that the error in the stiff component is greatly reduced and comparable to the error committed by the Gauss-Legendre method. However, the 
nonstiff component seems to suffer from round-off error. A full analysis of these methods warrants further investigation.

As explained at the start of Section 2, our interest lies in the stability analysis for travelling waves for the reaction-diffusion equation (10). By energy estimates similar to those in Brin [5, §3.2], we find that the eigenvalues are contained in the wedge given by

$$
\begin{gathered}
\operatorname{Re} \lambda \leq \frac{1}{4} c^{2}+\max _{\xi}\left|f^{\prime}(\hat{u}(\xi))\right|, \\
\operatorname{Re} \lambda+|\operatorname{Im} \lambda| \leq c^{2}+\max _{\xi}\left|f^{\prime}(\hat{u}(\xi))\right| .
\end{gathered}
$$

For the Fisher equation used as an example in the previous section, we have $\max _{\xi}\left|f^{\prime}(\hat{u}(\xi))\right|=1$. Therefore, the analysis reported in this paper is of limited use when assessing the stability of the travelling wave for this equation. However, for other equations the wedge (47) and the regime in which our analysis is valid may overlap.

We mentioned in the introduction that this work was instigated by the paper of Aparicio, Malham and Oliver [3. That paper studies a similar equation in the regime $\arg \lambda=\pi$, while the analysis in the current paper concerns the complementary regime $|\arg \lambda|<\pi-\varepsilon$ with $\varepsilon>0$. In fact, it is possible to derive a combined estimate for the local error valid in both regimes using WKB-analysis.

Finally, we wish to stress that the analysis reported here is only valid for scalar reaction-diffusion equations. We have not done a full analysis for systems of reaction-diffusion equations of the form (11). However, numerical experiments suggest that also in this case, the Magnus method suffers from order reduction but recovers the full order when matching the solutions to evaluate the Evans function. Generally, the Gauss-Legendre method still outperforms the Magnus method when $\lambda$ is away from the essential spectrum, but the difference is not so pronounced.

\section{ACKNOWLEDGEMENTS}

We would like to thank Arjen Doelman, Marco Marletta, Per Christian Moan and Khaled Saad for stimulating discussions during the preparation of this paper. We also thank the anonymous referees for their comments and suggestions for improvement of the manuscript.

\section{REFERENCES}

1. A. L. Afendikov and T. J. Bridges, Instability of the Hocking-Stewartson pulse and its implications for three-dimensional Poiseuille flow, Proc. R. Soc. Lond. A 457 (2001), 257-272. MR.1848089 (2002e:76024)

2. J. Alexander, R. Gardner, and C. Jones, A topological invariant arising in the stability analysis of travelling waves, J. Reine Angew. Math. 410 (1990), 167-212. MR.1068805 (92d:58028)

3. N. D. Aparicio, S. J. A. Malham, and M. Oliver, Numerical evaluation of the Evans function by Magnus integration, BIT 45 (2005), 219-258. MR2176193 (2006j:65158)

4. T. J. Bridges, G. Derks, and G. Gottwald, Stability and instability of solitary waves of the fifthorder KdV equation: A numerical framework, Physica D 172 (2002), 190-216. MR.1946769 (2004i:37148)

5. L. Q. Brin, Numerical testing of the stability of viscous shock waves, Ph.D. thesis, Indiana University, 1998.

6. - Numerical testing of the stability of viscous shock waves, Math. of Comput. 70 (2000), no. 235, 1071-1088. MR1710652 (2001j:65118)

7. P. J. Davis and P. Rabinowitz, Numerical integration, Blaisdell, 1967. MR0211604 (35:2482) 
8. I. Degani and J. Schiff, RCMS: Right correction Magnus series approach for integration of linear ordinary differential equations with highly oscillatory solution, J. Comput. Appl. Math 193 (2006), no. 2, 413-436. MR2229552

9. K. Eng $\varnothing$, On the construction of geometric integrators in the RKMK class, BIT 40 (2000), no. 1, 41-61. MR1759033 (2001c:65081)

10. J. W. Evans, Nerve axon equations: IV The stable and unstable pulse, Indiana Univ. Math. J. 24 (1975), no. 12, 1169-1190. MR0393891 (52:14698)

11. M. Facão and D. F. Parker, Stability of screening solitons in photorefractive media, Phys. Rev. E 68 (2003), no. 1, 016610.

12. R. A. Fisher, The wave of advance of advantageous genes, Ann. Eugenics 7 (1937), 353-369.

13. C. González, A. Ostermann, and M. Thalhammer, A second-order Magnus integrator for nonautonomous parabolic problems, J. Comput. Appl. Math 189 (2006), 142-156. MR2202970 (2006j:65269)

14. L. Greenberg and M. Marletta, Numerical solution of non-self-adjoint Sturm-Liouville problems and related systems, SIAM J. Numer. Anal. 38 (2001), no. 6, 1800-1845. MR1856233 (2002g:65086)

15. V. Gubernov, G. N. Mercer, H. S. Sidhu, and R. O. Weber, Evans function stability of non-adiabatic combustion waves, Proc. R. Soc. Lond. A 460 (2004), no. 2048, 2415-2435. MR:2073926 (2005f:80012)

16. E. Hairer and G. Wanner, Solving ordinary differential equations II. Stiff and differentialalgebraic problems, second ed., Springer-Verlag, Berlin, 1996. MR.1439506 (97m:65007)

17. M. Hochbruck and C. Lubich, On Magnus integrators for time-dependent Schrödinger equations, SIAM J. Numer. Anal. 41 (2003), no. 3, 945-963. MR2005189 (2004g:65089)

18. A. Iserles, A first course in the numerical analysis of differential equations, Cambridge University Press, 1996. MR $1384977(97 \mathrm{~m}: 65003)$

19. - On the global error of discretization methods for highly-oscillatory ordinary differential equations, BIT 42 (2002), no. 3, 561-599. MR1931887(2003i:65056)

20. A. Iserles, H. Z. Munthe-Kaas, S. P. Nørsett, and Antonella Zanna, Lie-group methods, Acta Numerica 9 (2000), 215-365. MR 1883629 (2003a:37123)

21. L. Jódar and M. Marletta, Solving ODEs arising from non-selfadjoint Hamiltonian eigenproblems, Adv. Comput. Math. 13 (2000), 231-256. MR.1772541 (2001m:65091)

22. T. Kapitula and B. Sandstede, Edge bifurcations for near integrable systems via Evans function techniques, SIAM J. Math. Anal. 33 (2002), no. 5, 1117-1143. MR1897705|(2003c:37109)

23. A. Kolmogorov, I. Petrovsky, and N. Piskunov, Étude de l'équation de la diffusion avec croissance de la quantité de matiére et son application à un probléme biologique, Bull. Univ. d'État à Moscou, Série Internationale, A 1 (1937), 1-25.

24. W. Magnus, On the exponential solution of differential equations for a linear operator, Comm. Pure and Appl. Math. 7 (1954), 639-673. MR0067873 (16:790a)

25. P. C. Moan, Efficient approximation of Sturm-Liouville problems using Lie-group methods, Tech. Report 1998/NA11, DAMTP, University of Cambridge, UK, 1998.

26. P. C. Moan and J. Niesen, Convergence of the Magnus series, Submitted, 2006. arXiv:math/0609198

27. C. Moler and C. van Loan, Nineteen dubious ways to compute the exponential of a matrix, twenty-five years later, SIAM Review 45 (2003), no. 1, 3-49. MR.1981253 (2004d:15012)

28. J. D. Murray, Mathematical biology, Springer, 1989. MR1007836 (90g:92001)

29. J. Niesen, On the global error committed when evaluating the Evans function numerically, Tech. Report HWM 06/43, Dept of Mathematics, Heriot-Watt University, 2006. arXiv:math/0611855

30. R. L. Pego and M. I. Weinstein, Eigenvalues, and instabilities of solitary waves, Philos. Trans. R. Soc. Lond. A 340 (1992), no. 1, 47-94. MR1177566 (93g:35115)

31. A. Prothero and A. Robinson, On the stability and accuracy of one-step methods for solving stiff systems of ordinary differential equations, Math. of Comput. 28 (1974), 145-162. MR0331793 (48:10125)

32. J. D. Pryce, Numerical solution of Sturm-Liouville problems, Clarendon Press, Oxford, 1993. MR.1283388 (95h:65056)

33. B. Sandstede, Stability of travelling waves, Handbook of Dynamical Systems II (B. Fiedler, ed.), North-Holland, 2002, pp. 983-1055. MR1901069 (2004e:37121) 
34. J. Swinton and J. Elgin, Stability of travelling pulse solutions to a laser equation, Phys. Lett. A 145 (1990), no. 8-9, 428-433. MR.1052867 (91c:81190)

35. D. Terman, Stability of planar wave solutions to a combustion model, SIAM J. Math. Anal. 21 (1990), no. 5, 1139-1171. MR1062397 (91f:35138)

Department of Mathematics, Heriot-Watt University, Edinburgh EH14 4AS, United KINGDOM

E-mail address: simonm@ma.hw.ac.uk

Department of Mathematics, Heriot-Watt University, Edinburgh EH14 4AS, United KINGDOM

Current address: Mathematics Department, La Trobe University, Victoria 3086, Australia

E-mail address: j.niesen@latrobe.edu.au 